\title{
On the Meaning of Concepts in Science Education
}

\section{Kostas Kampourakis ${ }^{1}$}

Published online: 5 October 2018

(C) Springer Nature B.V. 2018

Scientific concepts can be described as systematic mental representations of the natural world, and they have a central place and role in science. They may correspond to observable entities (e.g., "mammal" or "mountain"), to unobservable entities (e.g., "atom" or "gene"), or they can be related to processes (e.g., "photosynthesis" or "adaptation"). As a result, any kind of discourse about science has to involve concepts, the meaning of which ought to be clear among those participating in the discourse. However, this should not be taken as self-evident neither in science education nor in science itself. In contrast, ambiguity is possible because of features that are inherent in the concepts themselves. There are at least three issues to keep in mind: (1) that the meaning of particular terms in the vernacular language is different from the respective scientific concepts; (2) that many of the scientific concepts are, or relate to, metaphors; and (3) that the same scientific concept can have different meanings in different scientific contexts. Let us consider these issues one by one.

The first issue has to do with the vernacular meaning of words. For instance, the term "adaptation" in the everyday sense of the term refers to the adjustment of something to fit something else, e.g., to adapt a play form an original version in order to make it suitable for children. In most cases of the vernacular use of the term, adaptation relates to a conscious process of change that takes place in order for something to become suitable for specific conditions or uses. This notion is very different from the scientific concept of adaptation, which refers to an unconscious process of change through which populations, not individuals, adapt because the individuals with a specific trait that confers an advantage in the particular environment outnumber those that do not have it (these advantageous traits are also described as adaptations). Therefore, in order for students to understand the scientific concept of adaptation, teachers not only need to clearly explain the scientific meaning that has no connection to intentions, but also contrast it to the vernacular one that is related to intentionality. Otherwise, it is possible for students to extend the vernacular sense of the term, with which they are likely familiar, to its scientific use and think that adaptation is an intentional process.

A second issue relates to the use of metaphors. Metaphors are very common in science because in order to represent or explain something we do not fully understand, it is often easier

Kostas Kampourakis

Kostas.Kampourakis@unige.ch

1 Section of Biology and IUFE, University of Geneva, Geneva, Switzerland 
to refer to something else that is considered to have similar characteristics. For instance, cell biologists refer to "signaling" proteins and to "receptor" proteins to describe their chemical interactions that bring about changes in the status of a cell. Whereas the two proteins simply interact chemically, they are described as "signaling" and "receptor" because the outcome of this interaction is a change, or a series of changes, within the cell. Therefore, scientists describe the protein that makes the difference in bringing about these changes as a "signaling" protein, as if this protein is transferring a signal for initiating these changes. They also describe the other protein as a receptor because it is as if it receives the signal transferred by the other protein. Now, even though this may seem clear and straightforward, there can be problems in understanding. Students might for instance think that these proteins have the goal to fulfill their role in some conscious manner. However, not only these proteins do not have any intentions but also their interactions and subsequent changes depend on several other molecules around them. The problem here is that one might focus too much on the signal-receptor interaction and misunderstand, or even ignore, the broader cellular context in which the chemical interactions between these two proteins take place. The reception of the signal is something that humans perceive and use to describe the process, but not something that actually occurs. The use of these terms is metaphorical.

The last issue has to do with the meaning of a given concept in different subdisciplines. For example, population geneticists might focus on the frequencies of particular alleles in particular populations, and refer to the respective genes as factors that affect or are related to some phenotypic trait. In contrast, molecular biologists might refer to these very same genes as DNA segments that encode the information for the production of a molecule (protein in RNA) simply because they are interested in these or other related molecular processes. Because the referents in these definitions might, but do not have to, overlap, misunderstandings might occur. A factor that affects a trait can be related to a gene but it can also be related to a sequence outside genes. The gene concept of molecular biologists and population geneticists might thus not refer to the same entity. Therefore, it is important to clarify to what exactly one refers because different scientists might have in mind different meanings for the same concept. These different meanings must be made clear if we aim at conceptual understanding.

For all these reasons, it is necessary for science educators to look carefully into these issues, explain them to teachers, and refrain from taking for granted that the meaning of concepts is clear. Ambiguities, inconsistencies, and variation in the meaning of concepts are always possible. Even though it is necessary for us to have a common language in order to communicate, and even though it is not possible to perform a conceptual analysis every time we talk about concepts, it is nevertheless crucial to be aware of the related ambiguities, inconsistencies, and variation. Perhaps it is time to carefully reconsider the meaning of concepts in science education, in order to ensure a deep understanding of them and a lucid discourse with them.

We therefore welcome manuscripts, informed by the history and philosophy of science, which provide in-depth analyses of scientific concepts and discuss implications for science teaching and learning. It might be useful to think again about the meaning of concepts in science education.

\section{Compliance with Ethical Standards}

Conflict of Interest The author declares no conflict of interest. 